\title{
The integration of size and weight cues for perception and action Evidence for a weight-size illusion
}

\author{
Journal Article \\ Author(s): \\ Hirsiger, Sarah; Pickett, Kristen; Konczak, Jürgen \\ Publication date: \\ 2012-11 \\ Permanent link: \\ https://doi.org/10.3929/ethz-b-000057687 \\ Rights / license: \\ In Copyright - Non-Commercial Use Permitted \\ Originally published in: \\ Experimental Brain Research 223(1), https://doi.org/10.1007/s00221-012-3247-9
}




\title{
The integration of size and weight cues for perception and action: evidence for a weight-size illusion
}

\author{
Sarah Hirsiger $\cdot$ Kristen Pickett $\cdot$ Jürgen Konczak
}

Received: 19 November 2011/Accepted: 24 August 2012/Published online: 12 September 2012

(C) Springer-Verlag 2012

\begin{abstract}
Humans routinely estimate the size and weight of objects. Yet, when lifting two objects of equal weight but different size, they often perceive the smaller object as being heavier. This size-weight illusion (SWI) is known to have a lesser effect on motor control of object lifting. How the nervous system combines "weight" and "size" cues with prior experience and whether these cues are differentially integrated for perception and sensorimotor action is still not fully understood. Therefore, we assessed not only whether the experience of size biases weight perception, but also if experience of weight biases the size perception of objects. Further, to investigate differences between perceptual and motor systems for cue-experience integration, participants haptically explored the weight of an object with one hand and then shaped the aperture of their other hand to indicate its perceived size. Results-First, next to a SWI, healthy adults $(N=21)$ perceived lighter objects as being smaller and heavier objects as being larger, demonstrating a weight-size illusion (WSI). Second, participants were more susceptible to either the SWI or WSI. Third, aperture of the non-exploring hand was scaled to perceived weight and not to physical size. Hand openings were consistently smaller than
\end{abstract}

S. Hirsiger · K. Pickett - J. Konczak

Human Sensorimotor Control Laboratory, University

of Minnesota, Minneapolis, MN, USA

\section{S. Hirsiger}

Institute of Human Movement Sciences and Sport,

ETH Zürich, Zurich, Switzerland

\section{S. Hirsiger $(\square)$}

International Normal Aging and Plasticity Imaging Center, University of Zürich, Sumatrastrasse 30, 8006 Zurich,

Switzerland

e-mail: s.hirsiger@inapic.uzh.ch physical size, with SWI-sensitive participants being significantly more affected than WSI-sensitive subjects. We conclude: first, both size and weight perceptions are biased by prior experience. Weight perception is biased by expectations of size, while size perception is influenced by the expectancy of weight. Second, humans have the tendency to use one cue predominantly for both types of perception. Third, combining perceived weight with expected size influenced hand motor control, while online haptic feedback was largely ignored. Finally, we present a processing model underlying the size-weight cue integration for the perceptual and motor system.

Keywords Hand · Haptic perception - Human - Motor adaptation $\cdot$ Sensorimotor integration $\cdot$ Size perception

\section{Introduction}

Object manipulation requires the appropriate scaling of the hand aperture to the size of the object and the generation of lift and grip forces according to object weight. To estimate object properties and to issue appropriate motor commands in light of sensory and motor system noise, the central nervous system (CNS) combines afferent sensory cues with previous knowledge about object features. Recently, Bayesian Decision Theory has been applied to formalize processes of cue and sensorimotor integration (Körding and Wolpert 2006; Van Beers et al. 2002). According to this view, the nervous system combines prior knowledge about object properties gained through former experience ("the prior") with current sensory cues ("the likelihood"), to generate appropriate object property estimations ("the posterior") for action or perception. 
In most cases, the combination of prior and likelihood leads to an appropriate perception and behavior, but perception can be "misleading," if the prior is weighted higher than the likelihood, thus giving rise to a perception that does not correspond to the actual physical properties of the object. The size-weight illusion (SWI), first described by Charpentier (1891), represents such a case. The SWI arises when an individual lifts two objects of equal weight, but of different size, and perceives the smaller object as heavier. The illusion is robust (Chouinard et al. 2009; Dijker 2008; Flanagan et al. 2008; Flanagan and Beltzner 2000; Grandy and Westwood 2006; Kawai 2002, 2003; Murray et al. 1999), and the effect does not cease when the lifter is made aware that both objects have the same weight (Flanagan and Beltzner 2000). The SWI is believed to be a primarily haptic phenomenon as vision does not substantially contribute to the effect (Ellis and Lederman 1993).

There is evidence that perceptual and motor systems are differentially affected by the SWI. When people were asked to lift objects in an SWI context, they learned within the first 10 trials to scale their grip force according to the actual weight of the object, even though they still perceived the smaller object as heavier (Flanagan and Beltzner 2000). A subsequent study showed that experience reduces the prevalence of the SWI (Flanagan et al. 2008). Participants had trained up to 11 days to lift blocks with weights that varied inversely with volume. In addition to the rapid motor adaptation, they learned to predict the correct weights within about 240 lifts indicating that the perceptual system does adapt to weight cues, albeit at a slower pace than the motor system. Recent evidence suggests that these differences of the perceptual and motor system may be based on different mechanisms for integrating prior and likelihood (Brayanov and Smith 2010). The sensorimotor system is believed to function in a Bayesian manner when integrating probabilities subsequently used for motor commands. In contrast, the perceptual system integrates the prior and likelihood in an anti-Bayesian manner, overstating the value of unexpected sensory information (We will consistently use the term prior to refer to prior perceptual experience).

The size-weight illusion paradigm has been extensively studied to gain insight into the mechanism of cue integration for the perception of heaviness. However, to our knowledge, no study has investigated how the brain estimates object size based on expectations about weight. Thus, the aim of the present study was to investigate whether size perception is based on similar processes of prior and likelihood integration as the perception of heaviness. An experiment was designed in which participants lifted boxes of equal size but different weights, testing whether this would induce a weight-size illusion (WSI). We then sought to determine the differential effect of such an illusion on sensorimotor control using a size matching task. Finally, to obtain a standard of comparison that allowed us to assess the frequency and magnitude of a weight-size illusion, the same participants also lifted objects in a size-weight illusion context.

\section{Methods}

\section{Participants}

Twenty-one adults (11 $\hat{\jmath}, 10$ o; mean age $22.7 \pm 3.3$ years), naïve to the purpose of the study and with no history of neurological impairments, participated in this study. 18 out of 21 subjects were right-handed, according to the Edinburgh Handedness Inventory (Oldfield 1971). All subjects gave voluntary informed consent prior to participating. The study was approved by the Institutional Review Board of the University of Minnesota.

Stimuli and procedure: size-weight illusion experiment

\section{Stimuli}

Fifteen rigid plastic boxes of equal height $(6 \mathrm{~cm})$ and width (4 cm) but varying length $(3-10 \mathrm{~cm}$ in $0.5 \mathrm{~cm}$ increments) were used (for the purpose of this paper, we use the term "size" to refer to the length of the box). The size $6.5 \mathrm{~cm}$ box served as the standard stimulus. The remaining 14 boxes served as comparison stimuli. The weight of all comparison stimuli and the standard stimulus was $350 \mathrm{~g}$. Five additional boxes weighing 200, 250, 300, 400, or $450 \mathrm{~g}$ all with a fixed length of $6.5 \mathrm{~cm}$ were used as distracters.

\section{Procedure}

Participants were seated on an adjustable chair in front of a table. The upper arm on the non-dominant side was held in a neutral position with the elbow flexed at $90^{\circ}$ and the hand placed on the edge of the table. The non-dominant hand was used to execute all lifts. The standard or the comparison box was then placed in front of the participant's resting hand by the experimenter. After an auditory signal ("Lift"), the participant grasped the box along its length axis, which varied between boxes, using a precision grip (tips of the thumb and the index finger). The arm movement consisted of elbow flexion and extension in the sagittal plane. Participants were instructed to lift vertically and not to tilt the object. This eliminated any object sizedependent differences in torque or inertial moments, which may influence weight perception (Flanagan and Beltzner 2000). Participants were instructed to perform the lift to a height of about $10 \mathrm{~cm}$ above the table, then to hold the box 
in this position for $2 \mathrm{~s}$ (hold phase) while rendering a verbal judgment about its perceived heaviness. Vision was occluded throughout the experiment. In advance of the experiment, participants performed practice lifts to experience grasping without vision.

Judgments were based on the magnitude estimation procedure (Stevens 1953; Zwislocki and Goodman 1980), where participants voice a number that best represents the perceived weight of the box. No constraints were placed on the value of the first number, stating the magnitude of the first perceived weight. However, the numbers stated in subsequent trials had to be proportional to the increase or decrease in perceived object weight (e.g., if the initial weight was judged as " 5 ," a weight perceived as twice as heavy needed to be judged as "10"). All participants underwent a short training exercise and practice session before the start of the trials to ensure that they understood the procedure. After the rating, the box was placed back on the table and the hand returned to the starting point. As soon as the box was replaced, the experimenter exchanged the previous test box with a new box. The time interval between stimuli presentation was held constant. For each trial, the number value assigned by the participant to the comparison box could have been smaller (i.e., perceived as lighter), larger (i.e., heavier), or the same as the number assigned to the standard box.

Each trial consisted of two lifts, one using the $6.5 \mathrm{~cm}$ standard box and one using a comparison box. The order of lifts was pseudorandom and assured that subjects never lifted the same object more than twice in a row. Each of the 14 comparison boxes was compared four times to the standard object. The mean of the numbers assigned for the four trials represents the perceived magnitude estimation (PME) for a given comparison box. In addition, five randomly placed distracter trials, with boxes of different weights, were included in the testing sequence but were not used in further analysis. Thus, each subject executed 61 trials resulting in a total of 122 lifts. Participants were given two breaks to maintain their concentration level.

Stimuli and procedure: weight-size illusion experiment

\section{Stimuli}

While the SWI experiment used boxes of varying length, but constant weight, this experiment used a set of eleven boxes of different weights, but of constant length. Their weight ranged from 100 to $600 \mathrm{~g}$ in $50 \mathrm{~g}$ increments with the $350 \mathrm{~g}$ box serving as standard stimulus. The remaining ten boxes served as comparison stimuli. The length of each box was fixed at $6.5 \mathrm{~cm}$, which was the size of the standard box used in the SWI experiment.
Five additional boxes were used as distracters, varying in length $(5,5.5,6,7$, and $7.5 \mathrm{~cm})$ but with an identical weight of $350 \mathrm{~g}$. All of the boxes had the same height and width $(6 \times 4 \mathrm{~cm})$.

\section{Procedure}

Participants performed the same lifting movements as in the SWI experiment with their non-dominant hand, but were asked to make magnitude estimations about the perceived size instead of the weight of the box. In addition to giving a verbal judgment, participants were asked to match the perceived size of the box with the index finger and the thumb of their dominant hand as if they would grasp the same box. This hand aperture (HA) gesture was executed while the non-dominant hand held the object, assuring that differences in memory processing could not account for possible differences in size perception.

Hand apertures were recorded using an optoelectronic motion capture system (Peak Motus) with a 120-Hz sampling frequency. Each recording lasted $5 \mathrm{~s}$. Infrared reflective markers were attached to the tips of index finger and thumb. Before each data collection, a baseline recording was collected in which the participants were asked to hold the standard box along its length dimension $(6.5 \mathrm{~cm})$ using the thumb and index finger of their dominant hand. This procedure was performed to determine the distance between finger and thumb markers during holding, which allowed us to account for individual differences in finger size.

Each trial consisted of two lifts (standard and comparison box) for a total of 40 trials (four trials for each comparison box). Order of the presentation (comparison or standard) was pseudorandom. Five additional distracter trials, with boxes of varying length, were randomly inserted into the trial sequence, which were not investigated afterward. Vision was occluded at all times and two breaks were provided to assure continued attention. All participants completed the SWI and the WSI experiment at a maximum of 10 days apart (range: 1-10 days; mean: $4.6 \pm 2.3$ days). The testing order was counterbalanced across all subjects.

\section{Measurements}

\section{Measuring the frequency of the size-weight illusion}

To obtain a measure of how susceptible an individual was to perceive an illusion, we followed the procedure described by Kawai et al. (2007) and first computed the percentage of possible responses (e.g., heavier, similar, and lighter) for each size (e.g., $50 \%$ heavier, $25 \%$ lighter, and 
$25 \%$ same for the $5.5 \mathrm{~cm}$ box). These individual percentages were then averaged across subjects (i.e., mean of $\%$ heavier, mean of $\%$ lighter, and mean of $\%$ same) for each comparison box (see Fig. 1). To obtain a measure of the frequency of the illusion for each subject, their respective percentage responses "heavier" and "lighter" were computed and subsequently averaged (see Fig. 2). In other words, an individual's frequency of the occurrence of an illusion reflected how often the participant did not respond "same," which would have been the correct answer. For simplicity and in accordance with Kawai et al. (2007), we use the term frequency of the illusion rather than the "frequency of the occurrence of the illusion" in this paper.

\section{Size-Weight Illusion}
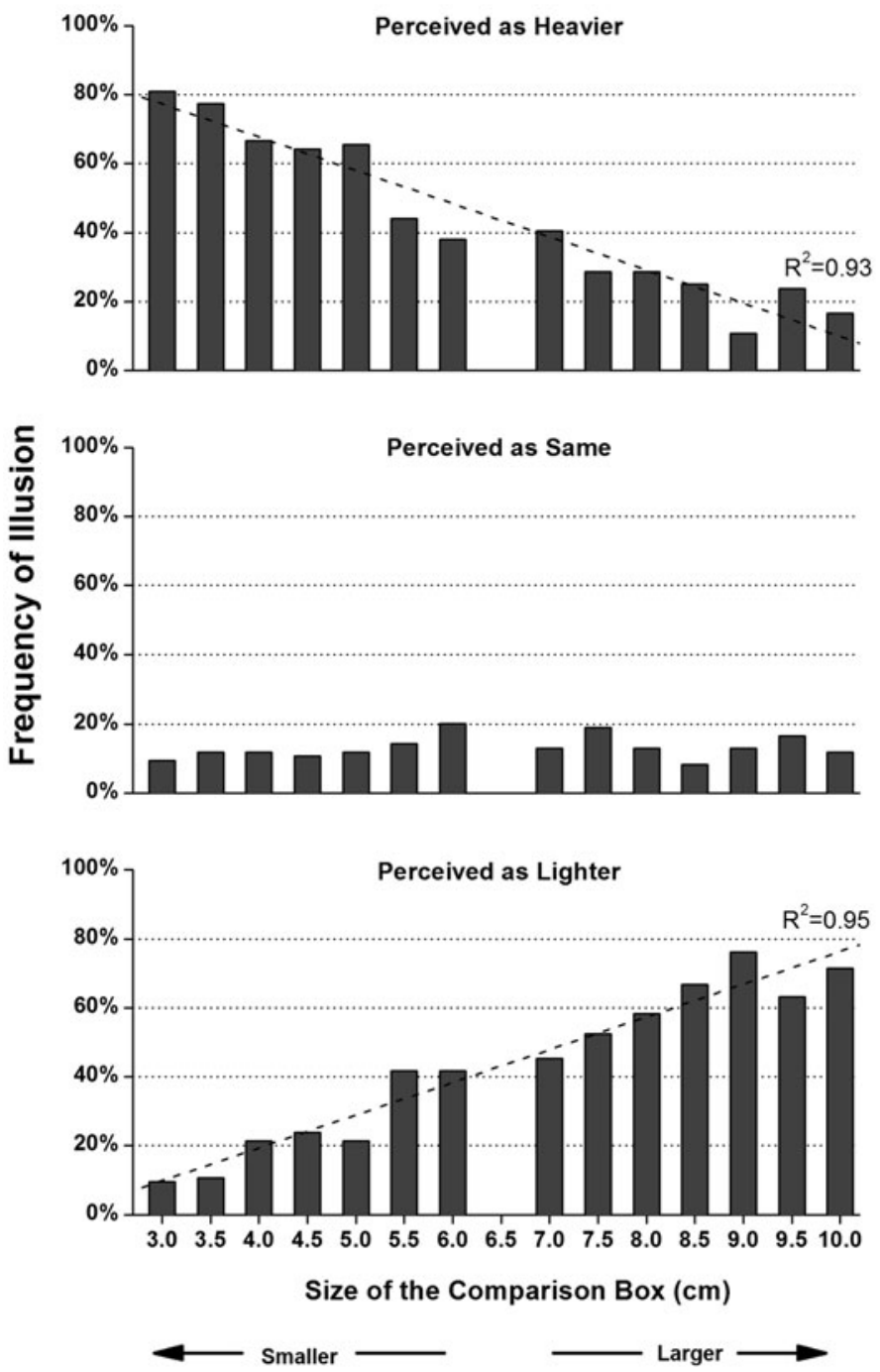

Fig. 1 Frequency of the size-weight illusion (SWI) and weight-size illusion (WSI) for each comparison box. Each bar represents the mean percentage of all participants' responses for each comparison box based upon their verbal magnitude estimation of either weight or size. SWI The shorter comparison boxes ranged from 3 to $6 \mathrm{~cm}$ and
Measuring the frequency of the weight-size illusion

To obtain the frequency of the occurrence of the WSI as a function of object weight, we employed the same procedure as described above. However, here the subjects' responses were categorized as either shorter (number assigned to the comparison box was smaller than the standard box) or longer (number assigned to the comparison box was larger than the standard box).

\section{Magnitude difference}

To correct for scaling shifts during the experiment, a perceived magnitude difference (PMD) between the

\section{Weight-Size Illusion}

Perceived as Shorter

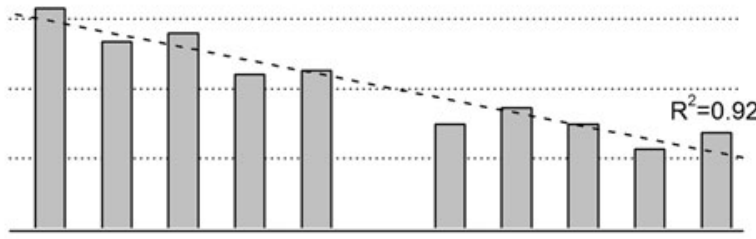

Perceived as Same

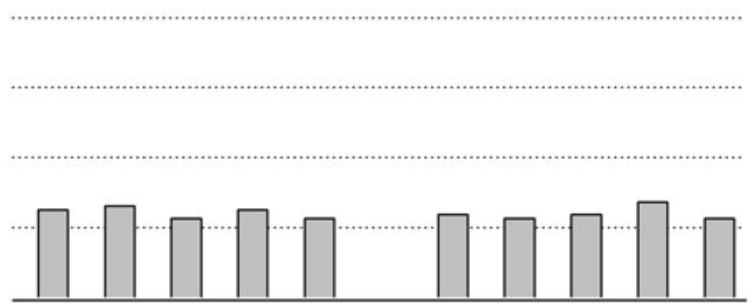

Perceived as Larger


the larger ranged from 7 to $10 \mathrm{~cm}$. The $6.5 \mathrm{~cm}$ box served as the standard. The weight for all boxes was $350 \mathrm{~g}$. WSI The lighter comparison boxes ranged from 100 to $300 \mathrm{~g}$ and the heavier from 400 to $600 \mathrm{~g}$. The size for all boxes was fixed at $6.5 \mathrm{~cm}$. The $350 \mathrm{~g}$ box served as the standard 


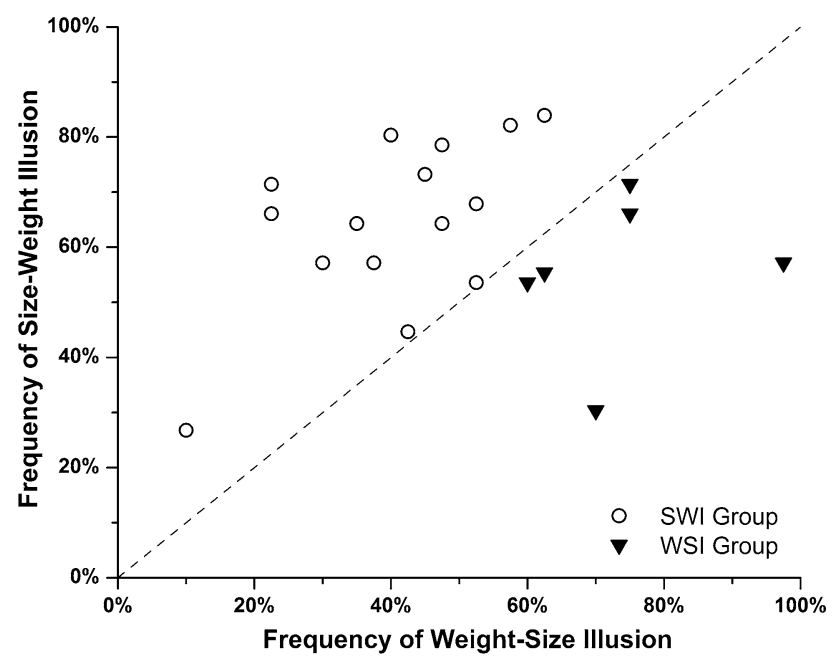

Fig. 2 Frequency of the size-weight illusion (SWI) and weight-size illusion (WSI) by participant. Each data point represents the observed frequency of the WSI and SWI for each participant. The dashed line indicates the line of equality, where both illusions occurred at equal frequency. Data points above the line of equality indicate that the participant was more susceptible to the SWI, while data points below the line of equality reflect a higher susceptibility to the WSI

comparison and the standard box in each trial was computed as follows:

$$
\begin{aligned}
\mathrm{PMD}= & {[(\mathrm{PME} \text { comparison box }-\mathrm{PME} \text { standard box }) /} \\
& \text { PME standard box }] * 100 \%,
\end{aligned}
$$

This conversion not only allows for comparison between first and last trials within each participant's data but also across participants. This ratio was further used to describe the strength of the illusions.

\section{Hand aperture measures}

The hand aperture data analysis was performed using customized software routines in MATLAB 7.0 Technical Programming Language. The raw time-position data of the markers were filtered offline using a fourth-order low-pass Butterworth filter with a cutoff frequency of $4 \mathrm{~Hz}$. From the $5 \mathrm{~s}$ recorded, the last second of the filtered time-position data (120 data points) was used to calculate the mean distance between the two markers, because the last second of holding the hand gesture most accurately reflected the final size judgment. If the last second was unusable due to dropping the scaling hand during the hold phase, a manually defined window of 120 data points was chosen to derive an aperture measure. The hand aperture displayed for each box was then calculated as follows, taking the calibration aperture (baseline recording) into account:
HA $=$ HA_Box $-($ Calibration Distance $-6.5 \mathrm{~cm})$,

with $6.5 \mathrm{~cm}$ indicating the size of the standard box, HA_Box $=$ hand aperture for the tested box and Calibration Distance $=$ size of the standard box + the dimension of the marked finger tips. The hand aperture data for each comparison stimuli and for the standard box were averaged for each participant across all trials. In addition to the HA, a hand aperture difference (HAD) was calculated for each comparison box using the following formula:

$$
\begin{aligned}
\mathrm{HAD}= & {[(\text { mean HA comparison box }- \text { mean HA standard box }) /} \\
& \text { mean HA standard box }] * 100 \%
\end{aligned}
$$

Normalization of data

Data from the two experiments could not be compared directly due to different units of the steps between the comparison boxes for the two experiments (cm vs. g). In order to make the two experiments comparable, each comparison box was labeled with a digit. The digit represented the number of steps the comparison box was apart from the standard box. Negative digits were assigned for comparison boxes, which were either smaller (SWI) or lighter (WSI). Comparison boxes, which were larger (SWI) or heavier (WSI) than the standard box, were given a positive digit. For the SWI, the positive labels for the larger boxes were: $1=7.0,2=7.5,3=8,4=8.5,5=9$, $6=9.5$, and $7=10 \mathrm{~cm}$. The smaller boxes were labeled as follows: $-1=6,-2=5.5,-3=5,-4=4.5$, $-5=4,-6=3.5$, and $-7=3 \mathrm{~cm}$. The same digits were used for the Experiment 2 (WSI). Heavier comparison boxes were therefore labeled as $1=400,2=450$, $3=500,4=550,5=600 \mathrm{~g}$ and $-1,-2,-3,-4$, and -5 were the labels for the $300,250,200,150$, and $100 \mathrm{~g}$, respectively. Thus, a change from $0.5 \mathrm{~cm}$ in the WSI was weighted as a 1 and was thus considered equal to a $50 \mathrm{~g}$ change in the SWI.

Data reduction and correction

The first three complete records of hand apertures for each comparison box and the first 30 records for the standard box were used for further analysis (first valid $75 \%$ of the recordings). If the minimum of three apertures for each comparison box was not obtained during the last second of recording, they were manually adjusted. Out of 1,140 valid apertures, 14 recordings were manually adjusted $(1.2 \%$ of the data). Due to technical problems with the motion capture system, hand aperture data of two participants were corrupted and could not be analyzed. 


\section{Results}

Evidence for a size-weight and a weight-size illusion

Participants showed a clear susceptibility for a haptically induced SWI. Despite the fact that all comparison boxes had the same weight, boxes which were smaller than the standard box were more frequently perceived as heavier, and conversely, comparison boxes larger than the standard box were perceived as lighter (Fig. 1). The frequency of the illusion decreased as the size difference between the comparison and the standard box became smaller. Illusion frequency for each individual participant ranged from 26.8 to $83.9 \%$ (mean $62.2 \pm 3.34 \%$ S.E.).

Participants were also susceptible to a WSI. Figure 1 shows the mean percentage of all participants shorter, or longer responses for each comparison box. Despite the fact that all comparison boxes had equal size $(6.5 \mathrm{~cm})$, participants perceived the size of individual boxes differently. Comparison boxes, which were lighter than the standard box, were more frequently perceived as shorter by the participants than the heavier comparison boxes. Heavier comparison boxes, in contrast, were perceived as longer. Illusion frequency decreased as the weight difference between the comparison and the standard box became smaller. WSI illusion frequency varied between participants ranging from 10.0 to $97.5 \%$ (mean $49.4 \pm 4.46 \%$ S.E.).

The data shown in Fig. 2 indicate that participants were differentially sensitive to either the SWI or the WSI. For further analysis, participants, who perceived the SWI more often than the WSI were assigned to a SWI-Group $(N=15)$, while participants with a higher frequency for WSI were categorized as the WSI-Group $(N=6)$. Subsequent regression procedures determined the respective slopes and intercepts (SWI group: FreqSWI $=39.5+0.63$ FreqWSI ( $\left.p_{\text {slope }}<0.001\right)$; WSI group: FreqSWI $=39.7+$ 0.22 FreqWSI $\left(p_{\text {slope }}=0.36\right)$.

Evidence for an illusion effect on hand aperture scaling

Participants scaled their hand opening according to expected size based on perceived weight and not according to the physical size of the box (which was a constant $6.5 \mathrm{~cm}$ ) (see Fig. 3). The respective mean hand apertures associated with holding the $100 \mathrm{~g}$ versus the $600 \mathrm{~g} \mathrm{com}$ parison box were significantly different for both groups (SWI-Group: $p<0.0001$; WSI-Group: $p<0.0001$ ) indicating that hand aperture-to-weight scaling was observed irrespective of differences in cue sensitivity. The data in Fig. 3 also indicate that both the WSI and SWI groups scaled their hand apertures consistently smaller than the physical size of the box across the tested range of weights, that is, participants tended to underestimate the true object size. Moreover, the amount of bias, that is the extent of underestimating size, was significantly larger in the SWI group when compared to the WSI group (WSI mean: $5.6 \mathrm{~cm} \pm 0.13$ S.E.; SWI mean: $4.9 \mathrm{~cm} \pm 0.07$ S.E.; $p<0.0001)$. Relating the observed aperture bias to physical object size showed that the hand aperture of the WSI group was $13.3 \%(0.9 \mathrm{~cm})$ smaller than the physical size, while the SWI-Group exhibited a bias of $25.5 \%(1.6 \mathrm{~cm})$.

To investigate whether participants adapted their hand aperture during repeated testing, the first and last hand aperture values for the 100 and $600 \mathrm{~g}$ comparison boxes were compared for each participant. No significant difference was found for either weight (100 g: SWI-Group: $p=0.415$, WSI-Group: $p=0.186 ; 600 \mathrm{~g}$ : SWI-Group $p=0.150$, WSI-Group $p=0.83$ ).

Comparison between perception and hand aperture scaling

Using perceived magnitude difference (PMD) and hand aperture difference (HAD) as relative measures allowed for a comparison of weight-size illusion effect on the perceptual versus the motor system. Both variables were highly correlated with each other $(r=0.88)$. This strong association between perceptual judgments and sensorimotor judgments was also observed when considering their respective raw measures (PME and $\mathrm{HA}, r=0.77$ ) that indicate size in absolute units $(\mathrm{cm})$, that is, the association is not explained by the transformation from absolute to

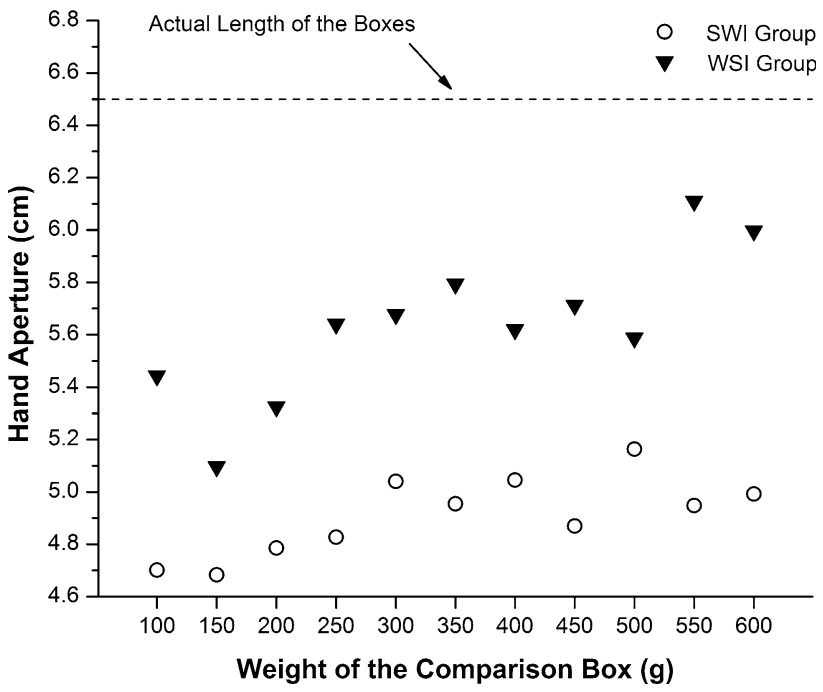

Fig. 3 Hand aperture as a function of physical weight of a box. The dashed line indicates the actual size of each box $(6.5 \mathrm{~cm})$. Data points represent the mean aperture across each group (SWI or WSI group). Note that aperture values were below the physical size in both groups with the magnitude of the underestimation being consistently smaller in the WSI-Group 
relative values. The PME mean values for each of the 10 comparison weights ranged from 4.99 to 6.04 , while the corresponding mean hand apertures ranged from 4.79 to $5.26(\mathrm{~cm})$ indicating that, on average, both perceptual as well as sensorimotor judgments underestimated physical object size $(6.5 \mathrm{~cm})$.

For the SWI-Group, the strongest illusion for the lighter comparison boxes was perceived for the $100 \mathrm{~g}$ box $(6.2 \mathrm{~cm}$ or $4.7 \%$ shorter). The $400 \mathrm{~g}$ box was perceived as the longest comparison box compared to the standard box (5.5\% longer). For the WSI-Group, the strongest illusions were perceived for the lightest and heaviest comparison boxes $(100$ and $600 \mathrm{~g})$. Converting the relative percentage scores to perceived size, the $100 \mathrm{~g}$ box was rated as $5.0 \mathrm{~cm}$ $(21.9 \%$ shorter) and the $600 \mathrm{~g}$ box as $8.3 \mathrm{~cm}(27.5 \%$ longer) than the standard, that is, there was a mean range of $3.3 \mathrm{~cm}$ between the smallest and largest perceived size for a box; both boxes had the same physical size $(6.5 \mathrm{~cm})$.

The relative difference data (PMD, HAD) of the weightsize illusion experiment revealed that both the perceptual and motor systems were influenced by the illusion (see Fig. 4). Respective linear regression procedures were computed to discern differences in slopes. For the SWIGroup, the slopes $(s)$ were not significantly different from each other (PMD: $s=0.014$, HAD: $s=0.015 ; p=0.83$; see Fig. 4 top), indicating that both systems were comparably biased by the different weights of the comparison boxes. In the WSI-Group, the respective regression coefficients for the slopes were different from each other (PMD: $s=0.093$, HAD: $s=0.023 ; p<0.0001$; see Fig. 4 bottom). However, when performing the equivalent analysis on the raw aperture data, this later difference is no longer present. Thus, caution is warranted in concluding a difference in gain between the two systems in the WSI group.

\section{Discussion}

This study was designed to achieve two aims: First, to obtain a better understanding of the underlying mechanisms of object perception by investigating how afferent feedback about size and weight and prior experience are combined by the perceptual system to achieve a stable percept. Second, to examine whether size and weight cues are differentially evaluated by the perceptual and sensorimotor system. Studying the saliency of size and weight as cues for perception and action in the context of illusions allowed for the systematic manipulation of either size or weight to examine the effect on perceptual as well as motor performance. The main results of the study were as follows: First, the study demonstrated the existence of a haptically induced weight-size illusion. This finding implies that size is not a dominant cue for both weight and
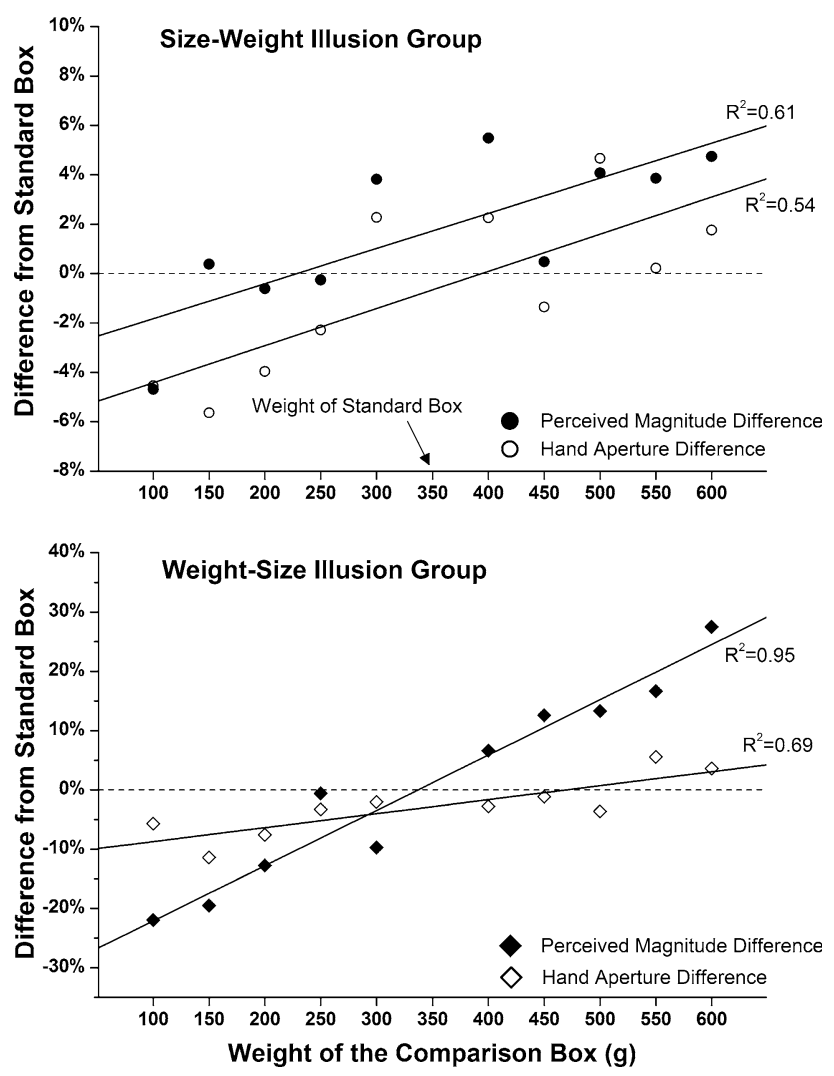

Fig. 4 Comparing perceptual and motor measures of object size as a function of object weight. Shown are the means for each subgroup (SWI vs. WSI-Group) when judging size as a function of weight. The abscissa shows the weight of the comparison boxes (standard $=350 \mathrm{~g}$ ), the ordinate represents the relative difference between the constant physical size $(6.5 \mathrm{~cm}$ for all boxes) and the verbal and motor-based size judgments (perceived magnitude difference vs. hand aperture difference). Note that ordinate scales differ between graphs as the WSI group exhibited larger differences in perceived magnitude than the SWI group

size perception. Instead, size and weight cues are both taken into account for both forms of perception. Second, not only weight but also size perception is based on an integration of both former experience and sensory input. Third, individuals had a tendency to be more sensitive to either weight or size cues, revealing individual differences in the way those cues were weighted during cue integration. Finally, perceived weight and not afferent haptic size information determined the aperture of the non-exploring hand, indicating that the motor system was influenced by the WSI.

The size-weight and the weight-size illusion

Ellis and Lederman (1993) concluded that the size-weight illusion is primarily a haptic phenomenon and that haptic cues alone had the same influence as the combination of haptic and visual cues on weight perception. Nevertheless, 
they also showed that SWI can be elicited by vision alone, although its effect is smaller. The current study replicated these earlier findings that haptic size cues alone can induce a size-weight illusion. As shown in Fig. 1, the frequency of the illusion increased as the difference between the comparison and standard box became larger in either direction, indicating a linear effect of haptic size cues on the perception of heaviness.

The primary focus of this study was to obtain a better understanding about size and weight cue integration and to examine whether and under what circumstances humans perceive a weight-size illusion. The influence from weight on volume perception had been studied before by Usnadze (1931). He reported that a tactile WSI can be elicited when objects of the same size, but different weights are placed on palmar surface of each hand with participants perceiving heavier objects as being smaller and lighter as being larger. We extended this tactile discrimination task to systematically investigate the influence of former experience about weight on the sensorimotor system. The results of the present study indicate the influence of previous experience about object weight on size perception, as participants perceived the lighter comparison boxes as shorter and the heavier as longer than the standard box (see Fig. 1). While this result seems to stand in contrast to the findings by Usnadze (1931), the differences in experimental procedure likely account for the different results. In the Usnadze experiment, object volume and not length was manipulated, and volume was assessed solely by placing the object on the surface of the palm, while participants actively grasped and lifted the object in our study. That is, in our paradigm, participants received tactile and proprioceptive signals as well as having access to predicted sensory feedback from the goal-directed action.

The WSI occurs through a combination of the experience-based expected size of the box (prior), and afferent sensory input about the object's size (likelihood). When the weight difference between the standard and comparison box increased, the WSI was more frequently perceived. This result is analogous to the SWI where the increasing difference between the sizes of the two boxes influenced weight perception.

Yet, our data also indicate that the integration of the prior and likelihood for weight and size perception may be based on different integration mechanisms. In the sizeweight illusion, participants perceived the smaller comparison box to be heavier than the standard box, but based on former experience, the smaller box should weigh less. This means that for weight perception, participants combined prior and afferent feedback in a manner contradictory to their experience (see Fig. 5). Brayanov and Smith (2010) referred to this as an anti-Bayesian form of integration.
Based on the experience, we would also expect a lighter box to be smaller and a heavier box to be larger. In the weight-size illusion, participants indeed perceived the lighter comparison boxes to be smaller and the heavier comparison boxes to be larger (see Fig. 1). This is consistent with the view that for size perception, participants combined prior and likelihood information in a Bayesian way of integration (see Fig. 5). The results from both illusion experiments therefore suggest that the nervous system uses different cue integration mechanisms for weight and size perception.

Evidence for different sensitivities to size-weight cues

Participants had a tendency to be more susceptible to either the SWI or the WSI. Given that participants were exposed to unimodal haptic size and weight cues in both experiments, the individual differences in the susceptibility to a particular illusion reflect differences in weight cue processing. Recent work demonstrated that humans exhibit different sensitivities to weight cues and that their individual weight cue sensitivity positively correlates with their susceptibility to the SWI (Kawai et al. 2007). Participants less sensitive to weight needed a larger difference between two weights to perceive them as dissimilar. The authors suggested that the illusion frequency for the sizeweight experiment is based on the difference between the standard and the comparison box and, at least partially, on differences in the sensitivity to weight cues. With respect to our findings, this interpretation implies that the difference in the frequency of the two illusions is due to individual differences in the sensitivity to either haptic size or weight cues. This differential cue susceptibility also had an effect on motor function, because those who were more susceptible to the WSI also had significantly larger hand openings to indicate perceived size that those more sensitive to weight cues (see Fig. 3).

\section{Using size cues for perception or sensorimotor action}

The assumption of two separate systems evaluating sensory information for perception and action is not new. The idea of two separate streams for processing visual information, the ventral and dorsal stream, was first described by Mishkin and Ungerleider (1982). In the framework of the Ebbinghaus illusion, Aglioti et al. (1995) reported that participants used the same hand aperture to grasp objects, which they had perceived as being different in size. Such dissociation between the motor and the perceptual system has also been observed within the context of the sizeweight illusion (Flanagan and Beltzner 2000; Grandy and Westwood 2006). These studies showed that grip force rapidly adapts to the physical weight of the box even 


\section{Perceptual System}

\section{Motor System}

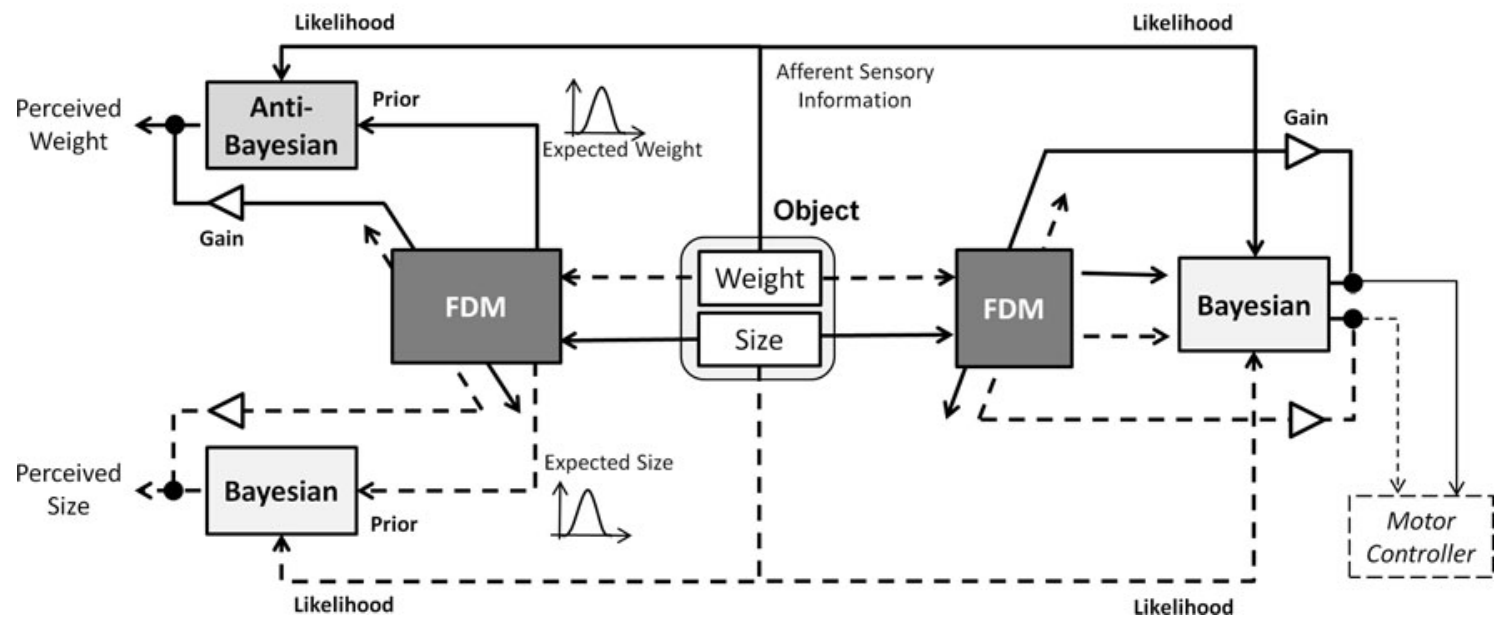

Fig. 5 Processing model of weight and size cues integration for action and perception. The object has two physical properties: weight and size. Solid arrows indicate processing flow for weight perception, while dashed arrows refer to size perception. For the perceptual and the motor system, the object's size and weight cues are inputs to a Forward Dynamic Model (FDM) representing the stored object experience. The FDM output is the experience-based expected weight or size, which can be described as a Gaussian distribution, and is referred to as the prior. The prior is then integrated with the likelihood, which represents the afferent sensory information about

though the participants still perceived the smaller object as heavier and the larger as lighter. Nevertheless, Flanagan et al. (2008) were able show that weight perception also adapts, but more slowly. Instead of five to ten trials, the perceptual system needs thousands of lifts. These findings may indicate that the perception prior differs from the action prior as it appears to be more resistant to change. The results of Brayanov and Smith (2010) indicate that although the prior and likelihood were integrated in an anti-Bayesian way for weight perception, the motor system seems to use a Bayesian integration method. These findings suggest a separation between the perceptual and sensorimotor system (i.e., grip force control was not guided by the lifter's perception of object weight-see Fig. 5). However, with respect to our data obtained in the WSI experiment, the strong correlation between sensorimotor and perceptual judgments of object size (hand aperture and verbal judgment) could also be interpreted as an indication that perceptual and sensorimotor systems at least share some information about object weight and size, that is, they may share neural resources and are not rigidly separated.

This study focused on the influence of the WSI on the motor system and how haptic size and weight cues are integrated. Our results show that the performance of the motor task was clearly influenced by varying the weights of the lifted boxes, as hand aperture increased as a function of increasing object weight (see Figs. 3, 4). The motor effect object weight and size. Two separate processing streams with two different integration mechanisms (Anti-Bayesian vs. Bayesian) are assumed for weight and size perception, respectively. A feedback loop with a specified gain provides the FDM with the final estimate of weight or size, which serves as a learning signal (arrows crossing the FDM boxes) for adapting object experience (e.g., when weight or size changes). Experimental findings in the context of the size-weight illusion and the weight-size illusion suggest separate cue integration processes for the perceptual and motor system

of the illusion persisted from the first lift to the last lift of a particular box as the scaling of the hand aperture did not change significantly over trials. Given that no feedback about performance was provided, the lack of an explicit error signal impeded learning.

However, this lack of adaptation during the WSI experiment allowed us to inspect how size cues are evaluated for sensorimotor action. If the sensorimotor system depends solely on afferent sensory information to adjust the opening of the hand to the size of an object, no variation in hand aperture for the different comparison boxes would have been observed, that is, hand aperture size would have been the same for all eleven boxes as all had the same physical size. This is not what we observed. In contrast, if the sensorimotor system only considers former experience (the prior) that size and weight increase linearly for boxes of the same density, then hand aperture should match the expected size for each weight. For example, if an object weighs a fourth of another object, the size of the lighter object should also be four times smaller than the size of the heavier object and the hand aperture should also be a fourth of the size of heavier box (i.e., $75 \%$ smaller). Yet, the smallest observed hand apertures were only $5 \%$ (SWI-Group) or $22 \%$ (WSI-Group) smaller than the standard box (see Fig. 4). These findings refute the assumption that the sensorimotor system relies solely on the prior to evaluate weight of a graspable object. Instead, 
it is consistent with a view that cue integration by the sensorimotor system combines both prior and likelihood in a Bayesian manner. Furthermore, our data show that the weighting of the prior and likelihood are dependent on the individual's sensitivity to either the weight or the size cues.

Finally, we need to consider that participants scaled their hand aperture consistently smaller than the physical size of the box across the tested range of weights (see Fig. 3), that is, while one hand explored the object, the other "matching" hand tended to underestimate true object size. Moreover, the amount of bias, that is the extent of underestimating size, was significantly larger in the SWI group when compared to the WSI group. The latter finding is explained by the individual differences in weight-size cue integration. However, the consistent underestimation cannot be explained as a sensory integration phenomenon. It is likely due to a bias introduced in subsequent sensorimotor transformations, that is, the processes necessary to transform perceived size to the motor commands of the opposite hand.

A model for integrating size and weight cues for action and perception

What does the finding of a weight-size illusion and its influence on the motor system mean for our understanding of cue integration for perception and sensorimotor action? Based on our results and previous work, it now becomes possible to arrive at a processing model that summarizes the differences in integration of sensory and prior information for perception and action (Fig. 5).

Consider first that during the lifting of a box, afferent haptic information about the object's weight and size becomes available centrally. Further assume that former object experience is stored in a Forward Dynamic Model (FDM) that captures the physical properties of the object. The input of the FDM is sensory cue information about size and weight, its output is the expected size or weight of the object. Thus, FDMs link size cues with expected weight and weight cues with expected size. The expected object features (weight/size) can then be described as a Gaussian distribution (given the system is not noise free) and can be referred to as the prior, representing the expectation based on former experience. One explanation for the occurrence of the SWI is a mismatch between the predicted sensory feedback generated by a forward dynamic model and the actual sensory feedback from the periphery (Ross 1969), although this sensorimotor mismatch hypothesis has been criticized (Ernst 2009), because it has been shown that the sensorimotor system adapts and corrects for this initial mismatch (Flanagan and Beltzner 2000).
The results from this study indicate that weight and size perception are based on the different combinations of prior and likelihood. For weight perception, the prior and the likelihood are integrated in an anti-Bayesian manner, overstating the value of unexpected sensory information. In contrast, for size perception, prior and likelihood are integrated in a Bayesian way. A feedback loop with a specified gain provides the FDM with a copy from the estimated weight enabling it to adapt to the actual physical weight/size of the lifted box. Based on the assumption that weight as well as size perception are based on the same FDM, the gain of the feedback loop has to be the same for the size as well as for weight perception.

The finding that the frequency of a particular illusion is related to an individual's varying sensitivity to weight or size is also explained by the model. For individuals who are more sensitive to size, the expected size (prior for size) has a higher perceptual weighting than the expected weight (prior for weight). The inverse would be true for an individual more sensitive to weight. Weighting one prior higher is only possible, if there is a separation at the level of integration (see Fig. 5).

Based on the findings of our and previous SWI experiments (Flanagan and Beltzner 2000; Grandy and Westwood 2006), a separation between processing object cues for perception and action is plausible. Here, the two systems only share the afferent sensory input (size and weight), but operate otherwise independently from each other. However, we need to be cognizant to the fact that this experiment cannot fully dissociate, if differences in scaling are the result of two separate neural processes (cue integration for perceptual or sensorimotor tasks) or are the result of one process with two distinct response continua. Nevertheless, the model can explain the existence of both size-weight illusions and provides a framework for the development of a more complete computational model.

\section{Conclusion}

The results of this study indicate that not only weight but also size perception are biased by former experience. In addition, the integration of prior and likelihood seems to be different for haptic size and weight perception (Bayesian vs. anti-Bayesian). Further, humans may be more susceptible to one specific haptic cue, either weight or size. Finally, the results of this study clearly indicate that both, the perceptual and the sensorimotor system, are biased by the WSI. However, in comparison with the SWI, it is not clear whether both systems work independently or if hand aperture was guided by the same processes that underlie size perception. 
Acknowledgments We sincerely thank Joshua Aman and Chiahao $\mathrm{Lu}$ from the Human Sensorimotor Control Laboratory for their technical support and help with the data collection.

\section{References}

Aglioti S, DeSouza JFX, Goodale MA (1995) Size-contrast illusions deceive the eye but not the hand. Curr Biol 5:679-685

Brayanov JB, Smith MA (2010) Bayesian and 'anti-Bayesian' biases in sensory integration for action and perception in the sizeweight illusion. J Neurophysiol 103:1518-1531

Charpentier A (1891) Analyse experimentale de quelques elements de la sensation de poids. Arch Physiol Norm Pathol 3:122-135

Chouinard PA, Large ME, Chang EC, Goodale MA (2009) Dissociable neural mechanisms for determining the perceived heaviness of objects and the predicted weight of objects during lifting: an fMRI investigation of the size-weight illusion. Neuroimage 44:200-212

Dijker AJ (2008) Why Barbie feels heavier than Ken: the influence of size-based expectancies and social cues on the illusory perception of weight. Cognition 106:1109-1125

Ellis RR, Lederman SJ (1993) The role of haptic versus visual volume cues in the size-weight illusion. Percept Psychophys 53:315-324

Ernst MO (2009) Perceptual learning: inverting the size-weight illusion. Curr Biol 19(1):R23-R25

Flanagan JR, Beltzner MA (2000) Independence of perceptual and sensorimotor predictions in the size-weight illusion. Nat Neurosci 3:737-741

Flanagan JR, Bittner JP, Johansson RS (2008) Experience can change distinct size-weight priors engaged in lifting objects and judging their weights. Curr Biol 18:1742-1747

Grandy MS, Westwood DA (2006) Opposite perceptual and sensorimotor responses to a size-weight illusion. J Neurophysiol 95:3887-3892
Kawai S (2002) Heaviness perception II. Contributions of object weight, haptic size and density to the accurate perception of heaviness or lightness. Exp Brain Res 147:23-28

Kawai S (2003) Heaviness perception IV. Weight $\times$ aperture -1 as a heaviness model in finger-grasp perception. Exp Brain Res 153:297-301

Kawai S, Henigman F, MacKenzie CL, Kuang AB, Faust PH (2007) A reexamination of the size-weight illusion induced by visual size cues. Exp Brain Res 179:443-456

Körding KP, Wolpert DM (2006) Bayesian decision theory in sensorimotor control. Trends Cogn Sci 10:319-326

Mishkin M, Ungerleider LG (1982) Contribution of striate inputs to the visuospatial functions of parieto-preoccipital cortex in monkeys. Behav Brain Res 6:57-77

Murray DJ, Ellis RR, Bandomir CA, Ross HE (1999) Charpentier (1891) on the size-weight illusion. Percept Psychophys 61:1681-1685

Oldfield RC (1971) The assessment and analysis of handedness: the Edinburgh inventory. Neuropsychologia 9:97-113

Ross HE (1969) When is a weight not illusory? Q J Exp Psychol 21:346-355

Stevens SS (1953) On the brightness of lights and loudness of sounds. Science 118:576

Usnadze D (1931) Über die Gewichtstäuschung und ihre Analoga [concerning the size-weight illusion and its analogues]. Psychol Forsch 14:366-379

Van Beers RJ, Baraduc P, Wolpert DM (2002) Role of uncertainty in sensorimotor control. Philos Trans R Soc Lond B Biol Sci 357:1137-1145

Zwislocki JJ, Goodman DA (1980) Absolute scaling of sensory magnitudes: a validation. Percept Psychophys 28:28-38 